\title{
The minimum number of monochromatic 4-term progressions in $\mathbb{Z}_{p}$
}

\author{
J. WOLF
}

\begin{abstract}
In this paper we improve the lower bound given by Cameron, Cilleruelo and Serra for the minimum number of monochromatic 4term progressions contained in any 2 -coloring of $\mathbb{Z}_{p}$ with $p$ a prime. We also exhibit a coloring with significantly fewer than the random number of monochromatic 4-term progressions, which is based on an a recent example in additive combinatorics by Gowers. In the second half of this paper we discuss the corresponding problem in graphs, which has received a great deal more attention to date. We give a simplified proof of the best known lower bound on the minimum number of monochromatic $K_{4}$ s contained in any 2-coloring of $K_{n}$ by Giraud, and briefly discuss the analogy between the upperbound graph constructions of Thomason and ours for subsets of $\mathbb{Z}_{p}$.
\end{abstract}

\section{Introduction}

Let $p$ be a prime. It is a pretty and well-known fact that in any 2-coloring of the cyclic group $\mathbb{Z}_{p}$ the number of monochromatic 3-term arithmetic progressions depends only on the densities of the color classes $R$ and $B$. Using discrete Fourier analysis, specifically the fact that $\widehat{1_{R}}(t)=-\widehat{1_{B}}(t)$ for $t \neq 0$, one easily obtains the result that the number of monochromatic 3 -term progressions in any coloring equals

$$
\frac{1}{2}\left(1-3 \alpha+3 \alpha^{2}\right) p^{2}
$$

where one of the color classes, $R$ say, has size $\alpha p$ (see also [4]). Note that this is precisely the number of 3 -term progressions we would expect if we were to choose the elements of the red color class independently at random from $\mathbb{Z}_{p}$ with density $\alpha$. Throughout this article, we shall be counting progressions without orientation, that is we shall be considering $3,5,7 \bmod 13$ as identical to 7, 5, 3 mod 13. Our results will be asymptotic in the order $p$ of the group.

While a similar formula holds for other equations in three variables, for example Schur triples of the form $x+y \equiv z$, this is not the case for longer 
progressions. It is not difficult to see that the number of monochromatic 4term progressions in a given 2-coloring does not just depend on the density ratio of the color classes. Instead, we will ask for the minimum number of monochromatic 4-APs in any 2-coloring of $\mathbb{Z}_{p}$, a quantity which we shall denote by $M_{4}(p)$. Bounding the more convenient normalized quantity $m_{4}=$ $2 M_{4}(p) / p^{2}$ is the aim we shall be concerned with throughout the first two sections of this paper.

An easy bound on $m_{4}$ can be derived from van der Waerden's Theorem. We know that the van der Waerden number $W(4)$ equals 35 , that is, any 2-coloring of 35 numbers in arithmetic progression is guaranteed to contain a monochromatic 4-AP. By averaging, we obtain a lower bound on $m_{4}$ of the form

$$
m_{4} \geq \frac{1}{185}+o(1)
$$

Here $o(1)$ denotes a quantity that tends to zero as $p$ tends to infinity through the primes.

This primitive estimate was significantly improved by Cameron, Cilleruelo and Serra [1] by observing that although the number 35 cannot be reduced when searching for monochromatic 4-APs, one only needs to color 7 points in arithmetic progression before one is guaranteed to find a monochromatic 4-AP or one which is evenly colored, i.e. one in which precisely 2 points are red and 2 points are blue. This fact together with one additional ingredient, which we shall inspect in more detail in Lemma 2.1 below, gives their bound

$$
m_{4} \geq \frac{1}{20}+o(1) .
$$

A further computational improvement yields their best effort of

$$
m_{4} \geq \frac{2}{33}+o(1) .
$$

In this paper we prove the following small improvement.

Theorem 1.1. Any 2-coloring of $\mathbb{Z}_{p}$ with $p$ a prime contains at least $p^{2} / 32$ monochromatic 4-term progressions, in other words,

$$
m_{4} \geq \frac{1}{16}+o(1) .
$$

In the other direction, it is clear that a random coloring with probability $1 / 2$ will contain $p^{2} / 16$ monochromatic 4 -APs, so that $m_{4} \leq 1 / 8+o(1)$. In Section 3 of this note we exhibit a coloring with fewer than this random 
The minimum number of monochromatic 4-term progressions in $\mathbb{Z}_{p} 55$

number of monochromatic 4-APs, which shows that the critical constant must lie strictly below $1 / 8$. More precisely, we shall prove the following theorem.

Theorem 1.2. There exists a 2-coloring of $\mathbb{Z}_{p}$ with fewer than (1$1 / 259200) p^{2} / 16$ monochromatic 4-term progressions, in other words,

$$
m_{4} \leq \frac{1}{8}\left(1-\frac{1}{259200}\right)+o(1) .
$$

We remark that while this paper concerns itself exclusively with the cyclic group $\mathbb{Z}_{p}$, the analogous question in the interval $\{1,2, \ldots, n\}$ is also of interest. Here the number of monochromatic 3-term progressions in a 2coloring does not simply depend on the density of the color classes, so even for the shortest progressions the question of determining the asymptotically minimal number is non-trivial and has not been resolved (for the best known upper and lower bounds see [9]). More general linear configurations are studied in [2].

It is clear from Theorems 1.1 and 1.2 that a gap between the upper and lower bound remains. Perhaps we shouldn't be too surprised at this state of affairs in view of the fact that the corresponding problem in graphs, where one wants to determine the minimum number of monochromatic $K_{4} \mathrm{~s}$ in any 2-coloring of the complete graph $K_{n}$, has resisted a complete resolution for quite some time and similar reasons. In Section 4 of this article, we give a simplified version of Giraud's argument [5] which yields the best known lower bound for this problem. In the final section we review some constructions by Thomason which yield 2-colorings of graphs with fewer than the random number of monochromatic $K_{4} \mathrm{~s}$ and discuss the analogy between graphs and sets. Perhaps the gap is accounted for by the different methods used to prove the upper and lower bounds in both cases: while the lower bounds are obtained by simple (if somewhat ingenious) counting, the upper bound constructions rely on Fourier analytic techniques.

\section{A lower bound on the minimum number of monochromatic 4-APs}

Given any 2-coloring $C$ of $\mathbb{Z}_{p}$, let $m_{4}(C)$ denote the number of monochromatic 4 -term progressions in $C$, divided by $p^{2} / 2$. For $i=0,1,2,3,4$, let $c_{i}=c_{i}(C)$ denote the number of 4 -term progressions in $\mathbb{Z}_{p}$ which have precisely $i$ red elements, divided by $p^{2} / 2$. We immediately note that $\sum_{i=0}^{4} c_{i}=1$ 
and $m_{4}(C)=c_{0}+c_{4}$. Write $E=c_{0}+c_{2}+c_{4}$ and $O=c_{1}+c_{3}$ for the normalized number of even- and odd-colored progressions, respectively.

A simple counting argument yields a further relation between the $c_{i}$ in terms of the density $\alpha$ of the red color class. The following lemma is borrowed from [1], although for the sake of self-containedness we give our own, more direct proof here.

Lemma 2.1. With the $c_{i}$ defined as above, we have that

$$
4\left(c_{0}+c_{4}\right)+\left(c_{1}+c_{3}\right)=4\left(1-3 \alpha+3 \alpha^{2}\right)
$$

for any coloring of $\mathbb{Z}_{p}$ in which the red color class has size $\alpha p$.

Proof. We will perform double-counting on the edges of a bipartite graph with vertex sets $X=X_{1} \cup X_{2}$ and $Y=Y_{1} \cup Y_{2} \cup Y_{3}$. Here $X_{1}$ consists of all 4 -APs counted by $c_{0}+c_{4}$, and $X_{2}$ of all those counted by $c_{1}+c_{3}$. $Y_{1}$ denotes the set of all monochromatic 3-APs, while $Y_{2}$ and $Y_{3}$ denote the sets of all monochromatic configurations of the form $x, x+d, x+3 d$ and $x, x+2 d, x+3 d$ respectively. Elements $x \in X$ and $y \in Y$ are joined by an edge if and only if $x$ contains the configuration $y$. It is now easy to see that the total (normalized) out-degree of $X$ equals $4\left(c_{0}+c_{4}\right)+\left(c_{1}+c_{3}\right)$, while the total out-degree of $Y$ equals twice the number of monochromatic 3 -APs plus the number of other monochromatic configurations in $Y$. By the first paragraph of the introduction, the number of such monochromatic 3 -term configurations equals $\left(1-3 \alpha+3 \alpha^{2}\right) p^{2} / 2$. Therefore the normalized out-degree of $Y$ equals $4\left(1-3 \alpha+3 \alpha^{2}\right)$.

The preceding lemma together with the identity $\sum_{i=0}^{4} c_{i}=1$ now implies that

$$
c_{0}+c_{4}=\frac{1}{3} c_{2}+\left(1-4 \alpha+4 \alpha^{2}\right) .
$$

Cameron, Cilleruelo and Serra immediately discard the second term on the right-hand side, which is indeed equal to zero for $\alpha=1 / 2$ and hence doesn't appear to be of much use. However, it will be vital for us to keep the dependence on the density of the red color class. From the above formula it is straightforward to see that any lower bound on the number of even-colored 4-APs (which we denoted by $E$ ) results in a lower bound on $m_{4}(C)$ via the formula

$$
m_{4}(C)=\frac{1}{2} E+\frac{3}{4}\left(1-4 \alpha+4 \alpha^{2}\right) .
$$

For the remainder of this section we shall aim to bound $E$ from below. In the process we shall focus on a method that works for densities close to 
$1 / 2$, since for densities bounded away from $1 / 2$ the second term in the lower bound for $m_{4}(C)$ already provides a fairly good estimate.

Given any 3 -term progression $S$, let $p_{S}$ denote the number of evenly colored 4-APs which contain $S$. Let $q_{S}$ be the number of 4-APs containing $S$ which are not evenly colored. It is obvious from these definitions that $0 \leq p_{S}, q_{S} \leq 2$ and $p_{S}+q_{S}=2$. Another second's thought confirms that

$$
\mathbb{E}_{S} p_{S}=2 E \text { while } \mathbb{E}_{S} q_{S}=2 O,
$$

where the expectation operator $\mathbb{E}_{S}$ denotes the sum $\sum_{S}$ divided by $p^{2} / 2$. Any 3 -term progression $S$ of the form $x, x+d, x+2 d$ determines a unique (unordered) pair of points $(a, b)$ such that the five points and each of the quadruples $a, x, x+d, x+2 d$ and $x, x+d, x+2 d, b$ lie in arithmetic progression. We shall call the pair $(a, b)$ a frame pair. It is straightforward to see that each frame pair belongs to a unique 3 -term progression. Note that in these statements we have used the assumption that $p$ is prime.

The crucial observation is that the two 4-APs containing $S$ have different color parities if and only if the frame pair of $S$ is bichromatic. Therefore, $p_{S}-q_{S}$ is not equal to zero if and only if $S$ has a monochromatic frame pair. For densities close to $1 / 2$, the total number of monochromatic pairs is at its minimum, which will enable us to get an acceptable estimate in this density regime. As remarked before, for densities bounded away from $1 / 2$ the second term in the lower bound for $m_{4}(C)$ will take over.

We find a trivial lower bound on $E$ of the form

$$
2 E=2 O+\mathbb{E}_{S}\left(p_{S}-q_{S}\right) \geq 2(1-E)-\mathbb{E}_{S}\left|p_{S}-q_{S}\right| .
$$

But $\mathbb{E}_{S}\left|p_{S}-q_{S}\right|$ precisely equals 2 times the appropriately normalized number of monochromatic pairs in the coloring. Now the number of monochromatic (unordered) pairs in a coloring of $\mathbb{Z}_{p}$ in which one color class has density $\alpha$ is precisely $\left(\alpha^{2}+(1-\alpha)^{2}\right) p^{2} / 2$, which yields

$$
E \geq \frac{1}{2}\left(1-\left(1-2 \alpha+2 \alpha^{2}\right)\right)=\alpha(1-\alpha),
$$

which in turns produces the bound

$$
m_{4}(C) \geq \frac{1}{4}\left(\alpha(1-\alpha)+3\left(1-4 \alpha+4 \alpha^{2}\right)\right) .
$$

The minimum of this function is easily seen to be $1 / 16$, attained at $\alpha=1 / 2$, concluding the proof of Theorem 1.1. The next section shows that we have found the correct bound at least to within a factor of 2 . 


\section{A colouring with few monochromatic 4-APs}

Very recently Gowers [7] gave an example of a uniform subset of $\mathbb{Z}_{p}$ which contains fewer than the number of 4-APs expected in a random subset of $\mathbb{Z}_{p}$ of the same density. By uniform we mean that the largest non-trivial Fourier coefficient of the indicator function of the set is $o(1)$ in modulus. It is easy to establish that uniform sets always contain the number of 3 term progressions expected in a random subset of $\mathbb{Z}_{p}$ of the same density. It had been known for quite some time that ordinary Fourier analysis was insufficient when it came to counting longer progressions. Indeed, it is not too difficult to construct uniform sets that contain significantly more than the expected number of progressions of length 4. It was Gowers's intention to show that it is possible to achieve a negative 4-AP count (compared with random) while retaining the uniformity of the set. (For progressions of length strictly greater than 4 this is significantly easier, see the remarks in [7].)

In this section we observe that this construction immediately gives rise to a 2-coloring of $\mathbb{Z}_{p}$ with strictly fewer than $p^{2} / 16$ monochromatic 4 -APs. For $A \subseteq \mathbb{Z}_{p}$, let $p_{4}(A)$ denote the number of 4 -term progressions in $A$, divided by $p^{2} / 2$, and let $m_{4}(A)$ denote the number of monochromatic 4 -APs in the coloring $C$ which is induced by $A$ (that is, we take $R=A$ and $B=A^{C}$ ), normalized in the same way. For uniform sets, the quantities $m_{4}(A)$ and $p_{4}(A)$ are related as follows.

Lemma 3.1. Given a uniform set $A \subseteq \mathbb{Z}_{p}$ of density $\alpha$, we have the relation

$$
m_{4}(A)=\frac{1}{2}\left((1-\alpha)^{4}-\alpha^{4}\right)+2 p_{4}(A)+o(1) .
$$

Proof. Writing $1_{A}$ for the characteristic function of the set $A$, we find that

$$
\begin{aligned}
2 m_{4}(A)= & \mathbb{E}_{x, d} 1_{A}(x) 1_{A}(x+d) 1_{A}(x+2 d) 1_{A}(x+3 d) \\
& +\mathbb{E}_{x, d} 1_{A^{C}}(x) 1_{A^{C}}(x+d) 1_{A^{C}}(x+2 d) 1_{A^{C}}(x+3 d) \\
= & 1-4 \alpha+6 \alpha^{2}+4 p_{4}(A) \\
& -\mathbb{E}_{x, d} 1_{A}(x) 1_{A}(x+d) 1_{A}(x+2 d) \\
& -\mathbb{E}_{x, d} 1_{A}(x+d) 1_{A}(x+2 d) 1_{A}(x+3 d) \\
& -\mathbb{E}_{x, d} 1_{A}(x) 1_{A}(x+d) 1_{A}(x+3 d) \\
& -\mathbb{E}_{x, d} 1_{A}(x) 1_{A}(x+2 d) 1_{A}(x+3 d) .
\end{aligned}
$$

It is easily seen that all the 3-term configurations appearing in the above sum appear in the expected number, by writing, for example, 
The minimum number of monochromatic 4-term progressions in $\mathbb{Z}_{p} 59$

$$
\mathbb{E}_{x, d} 1_{A}(x) 1_{A}(x+d) 1_{A}(x+2 d)=\sum_{t} \widehat{1_{A}}(t)^{2} \widehat{1_{A}}(-2 t)=\alpha^{3}+o(1),
$$

assuming that the subset $A$ is sufficiently uniform, that is $\sup _{t \neq 0}\left|\widehat{1_{A}}(t)\right|=$ $o(1)$.

We shall briefly sketch Gowers's construction with a slight numerical improvement over the original version. It is included for the sake of completeness and for purposes of comparison with the graphs case later on. We shall conclude the section with a statement of the exact bound we obtain.

Gowers starts off by constructing a function $g$ taking values \pm 1 on the cube $\{1,2,3,4\}^{3}$ which satisfies

$$
\sum_{x, d} g(x) g(x+d) g(x+2 d) g(x+3 d)=-72 .
$$

This is done on the basis of a geometric argument. He then proceeds to project the cube into the interval $[1,300]$ using a map $\phi$. (For the reader familiar with this kind of argument, the map $\phi$ is a standard Freiman isomorphism.) Next one defines a new function $f$, which takes values \pm 1 and 0 on the interval $[1,300]$, by setting $f(x)=g\left(\phi^{-1}(x)\right)$ if $x$ lies in the image of the projection $\phi$ of the cube, and $f(x)=0$ otherwise. By construction, $f$ also satisfies

$$
\sum_{x, d} f(x) f(x+d) f(x+2 d) f(x+3 d)=-72,
$$

that is, it has negative relative 4-AP count. Elegant and neat as the geometric example is, it is also rather inefficient. By an exhaustive numeric search for small examples we found the interval $[1,18]$, on which we let $f$ take successive values

$$
-1,-1,-1,1,-1,-1,1,-1,-1,-1,-1,1,1,1,-1,1,-1,-1
$$

and for which

$$
\sum_{x, d} f(x) f(x+d) f(x+2 d) f(x+3 d)=-36 .
$$

Due to our rather primitive programming methods we cannot rule out the existence of even more efficient small examples on intervals of length greater than 25 at this point. 
The next stage is to blow up this small example to one that lives inside $\mathbb{Z}_{p}$ for large $p$. To this end, we define, following Gowers, a function $F: \mathbb{Z}_{p} \rightarrow$ $\{-1,0,1\}$ by setting $F(x)=f(t)$ whenever $x \in I_{t}$, where $I_{t}$ stands for the interval $((2 t-1) m, 2 t m]$ and $m$ is a positive integer between $p /(5 \times 18)$ and $p /(4 \times 18)$. It is easy to check that $F$ is well-defined, and that the 4 -AP counts of $F$ and $f$ are related via

$\sum_{x, d} F(x) F(x+d) F(x+2 d) F(x+3 d)=s \sum_{x, d} f(x) f(x+d) f(x+2 d) f(x+3 d)$

where $s \geq m^{2} / 9$. It remains to ensure that $F$ is uniform, and to convert the \pm 1 function into a subset of $\mathbb{Z}_{p}$. The former is achieved by multiplying $F$ by an appropriate sum of quadratic exponentials, giving rise to a function $G: \mathbb{Z}_{p} \rightarrow[-4,4]$ defined by

$$
G(x)=F(x)\left(\omega^{x^{2}}+\omega^{3 x^{2}}+\omega^{-3 x^{2}}+\omega^{-x^{2}}\right),
$$

where $\omega$ is a $p$ th root of unity (note that the negative exponents are needed to make the resulting function $G$ real). Since $F$ essentially behaves like the indicator function of a union of intervals, its Fourier transform has bounded $\ell^{1}$-norm, and because of the large amount of cancellation coming from the quadratic phases we can conclude that all non-trivial Fourier coefficients of $G$ are tiny. A fairly tedious computation shows that the 4-AP count of $G$ is asymptotically twice that of $F$. Finally, turning the function $G$ into a subset of $\mathbb{Z}_{p}$ is a completely standard procedure in which, roughly speaking, we choose an element $x$ to lie in $A \subseteq \mathbb{Z}_{p}$ with probability $(4+G(x)) / 8$. It can be checked that with high probability the resulting set $A$ has density $1 / 2$ and is uniform by construction but contains at most

$$
\left(\frac{1}{16}-\frac{2 \times 36}{9(5 \times 18)^{2} 2^{12}}\right) p^{2}
$$

4-term progressions (up to lower order terms). In conjunction with Lemma 3.1, this discussion concludes our proof of Theorem 1.2.

Incidentally, it is also interesting to combine (via Lemma 3.1) this approach with the lower bound on $m_{4}$ we obtained in Section 2. It tells us that any uniform subset of density $1 / 2$ must contain at least $p^{2} / 644$-term progressions. This is related to a question Gowers asks in [7] and which can be traced back to I. Ruzsa: if $A \subseteq \mathbb{Z}_{p}$ is uniform of density $\alpha$, must $A$ contain at least $\alpha^{c}$ progressions of length 4 for some large constant $c$ ? Of course, for 
The minimum number of monochromatic 4-term progressions in $\mathbb{Z}_{p} 61$

densities bounded away from $1 / 2$ the considerations described here yield no results.

Although transferring results from the density to the coloring world and vice versa has proved fruitful in this instance, we very much doubt that the correct constant for the problem of counting the minimal number of monochromatic 4 -term progressions can be obtained in this way.

\section{Giraud's lower bound for the number of monochromatic $K_{4} \mathrm{~S}$}

In this section we give a simplified proof of Giraud's lower bound [5] on the minimum number of monochromatic $K_{4}$ s which we are guaranteed to find in any 2-coloring of the edges of the complete graph $K_{n}$ on $n$ vertices. We call this quantity $M_{K_{4}}(n)$, and its normalized sibling $m_{K_{4}}=M_{K_{4}}(n) /\left(\begin{array}{l}n \\ 4\end{array}\right)$. Since we are only concerned with asymptotics, we shall for the remainder take a rather relaxed approach to equalities: $x \asymp 1$ will always mean $x=1+o(1)$. We shall again be using the expectation operator $\mathbb{E}$, denoting the sum over edges or triangles normalized by $\left(\begin{array}{l}n \\ 2\end{array}\right)$ or $\left(\begin{array}{l}n \\ 3\end{array}\right)$, respectively.

As in the case of 4-APs, a simple lower bound can be given by averaging using Ramsey's Theorem. Giraud proved the much superior lower bound

$$
m_{K_{4}}>\frac{1}{46}+o(1)
$$

and we shall give a concise exposition of his work here, including a simplification of his argument. The original presentation in [5] is rather convoluted, and only available in French.

Throughout, we shall fix a coloring of $K_{n}$ and color-blindly count the following configurations on four vertices: the complete graph on 4 vertices denoted by $K$, the double triangle (or $K$ with one edge missing) $D T$, the triangle with a pendant edge $T E$, the 4 -cycle $C$, the ordinary triangle $T$ and the path of length 3 denoted by $P$. We shall abuse notation and use the acronyms to denote the number of occurrences of these structures divided by $\left(\begin{array}{l}n \\ 4\end{array}\right)$. We call the collection of these substructures $\mathcal{Q}$.
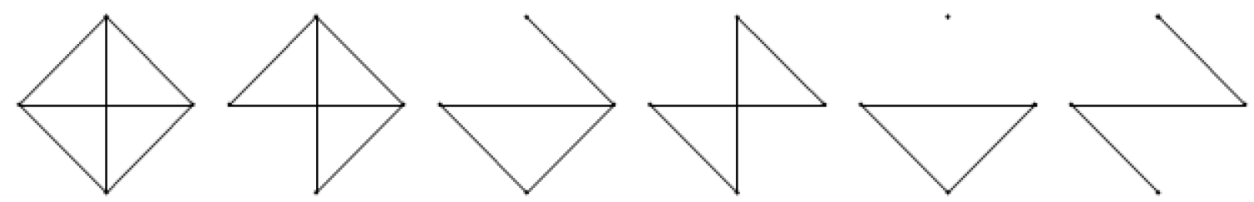

Following Giraud, we define for a given edge $e$ the quantities $m_{e}=\#$ monochromatic triangles containing $e$, divided by $n-2, b_{e}=$ normalized \# 
bichromatic triangles containing $e$ in which $e$ is the only edge of its color, and $c_{e}=$ normalized \# bichromatic triangles containing $e$ in which $e$ is not the only edge of its color.

We immediately see that $m_{e}+b_{e}+c_{e}=1$. Considering the number of mono- and bichromatic triangles in members of $\mathcal{Q}$, we find the following system of equations:

$$
\begin{aligned}
& 12 \mathbb{E}_{e}\left(\begin{array}{c}
m_{e} \\
2
\end{array}\right) \asymp 6 K+D T \\
& 12 \mathbb{E}_{e} m_{e} b_{e} \asymp T E+3 T \\
& 12 \mathbb{E}_{e} m_{e} c_{e} \asymp 4 D T+2 T E \\
& 12 \mathbb{E}_{e} b_{e} c_{e} \asymp 2 T E+4 P \\
& 12 \mathbb{E}_{e}\left(\begin{array}{c}
b_{e} \\
2
\end{array}\right) \asymp D T+2 C
\end{aligned}
$$

Thus, taking suitable linear combinations of these equations, we obtain

$$
\begin{aligned}
K+D T+T E+C+T+P & =1 \\
K+T E+C-(D T+T+P) \asymp & 32 K-3 \mathbb{E}_{e} b_{e}+11 \mathbb{E}_{e} m_{e} \\
& -48 \mathbb{E}_{e} m_{e}^{2}+6 \mathbb{E}_{e}\left(m_{e}-b_{e}\right)^{2}
\end{aligned}
$$

Still following Giraud, we define for given a triangle $t$ the parameters $p_{t}=$ \# even-edged configurations in $\mathcal{Q}$ containing $t$, divided by $n$, and $q_{t}=$ normalized \# odd-edged configurations in $\mathcal{Q}$ containing $t$. Double-counting again, we find that

$$
\mathbb{E}_{t} p_{t}=K+T E+C \quad \text { and } \quad \mathbb{E}_{t} q_{t}=D T+T+P
$$

Combining this with the two previous equations and using the fact that $\mathbb{E}_{e}\left(m_{e}+3 b_{e}\right)=1$, we obtain

$$
32 K=1-12 \mathbb{E}_{e} m_{e}+48 \mathbb{E}_{e} m_{e}^{2}-6 \mathbb{E}_{e}\left(m_{e}-b_{e}\right)^{2}+\mathbb{E}_{t}\left(p_{t}-q_{t}\right)
$$

We observe the similarities with our work in Section 2, and note the increased level of difficulty here due to the increased complexity of the substructures.

The remainder of the proof consists in bounding the final two terms above in modulus by a suitable application of the Cauchy-Schwarz Inequality, and then performing an optimization over what is essentially the mean and variance of the variables $m_{e}$. Before carrying out this plan we shift our variables by setting

$$
\mu_{e}=4 m_{e}-1 \quad \text { and } \quad \delta_{e}=2\left(m_{e}-b_{e}\right)
$$


With these definitions, the parameters with respect to which we optimize later are

$$
s=\mathbb{E}_{e}\left(\mu_{e}-\delta_{e}\right)=\frac{1}{3} \mathbb{E}_{e} \mu_{e}, \quad r=\mathbb{E}_{e}\left(\mu_{e}-\delta_{e}\right)^{2}, \quad p=\mathbb{E}_{e} \mu_{e}^{2} .
$$

It is now possible to rewrite equation (1) as

$$
32 K=1+3 \mathbb{E}_{e} \mu_{e}+3 \mathbb{E}_{e} \mu_{e}^{2}-\frac{3}{2} \mathbb{E}_{e} \delta_{e}^{2}+\mathbb{E}_{t}\left(p_{t}-q_{t}\right) .
$$

Our first task is to find an upper bound for $\mathbb{E}_{e} \delta_{e}^{2}$ : Let $B$ denote the number of bichromatic triangles in the graph $K_{n}$, and set $b=B /\left(\begin{array}{l}n \\ 3\end{array}\right)$. Using the fact that every non-monochromatic triangle is bichromatic, it is straightforward to compute that $b=3 / 4(1-s)$. We need some additional notation: For any vertex $i$, let $r_{i}$ denote the number of red edges incident with $i$, divided by $n-2$, and $b_{i}$ the normalized number of blue edges. For an edge $e=i j$, we let $c_{e}^{\prime}$ denote the number of bichromatic triangles including $e$ where the two edges of the same color meet in vertex $i$. Let $c_{e}^{\prime \prime}=c_{e}-c_{e}^{\prime}$. If the edge $e=i j$ is colored red, it is clear that $r_{i} \asymp m_{e}+c_{e}^{\prime}$ and $r_{j} \asymp m_{e}+c_{e}^{\prime \prime}$. Now the total proportion $b$ of bichromatic triangles can also be expressed as

$3 / 2 \mathbb{E}_{e=i j} r_{i} b_{i}+r_{j} b_{j} \asymp 3 / 2 \mathbb{E}_{e}\left(m_{e}+c_{e}^{\prime}\right)\left(1-\left(m_{e}+c_{e}^{\prime}\right)\right)+\left(m_{e}+c_{e}^{\prime \prime}\right)\left(1-\left(m_{e}+c_{e}^{\prime \prime}\right)\right)$,

the right-hand side of which can be bounded above by $3 / 2 \mathbb{E}_{e}\left(2 m_{e}+c_{e}\right)-$ $1 / 2\left(2 m_{e}+c_{e}\right)^{2}$. This in turn can be expressed in terms of $\delta_{e}$ to give the bound $\mathbb{E}_{e} \delta_{e}^{2} \leq 4 s$.

We now turn to bounding $\mathbb{E}_{t}\left(p_{t}-q_{t}\right)^{2}$. We note that, given a triangle $t$ and an edge $e=i j$, the structures induced by $t \cup i$ and $t \cup j$ have different color parities if and only if an odd number of edge pairs $(i v, j v)_{v \in t}$ differ in color. It follows that

$$
\mathbb{E}_{t} p_{t} q_{t} \asymp 3 \mathbb{E}_{e}\left(\left(\begin{array}{c}
c_{e} \\
3
\end{array}\right)+c_{e}\left(\begin{array}{c}
m_{e}+b_{e} \\
2
\end{array}\right)\right),
$$

which can be rearranged to give

$$
\mathbb{E}_{t}\left(p_{t}-q_{t}\right)^{2} \asymp \mathbb{E}_{e}\left(1-2 c_{e}\right)^{3} .
$$

The latter expectation can be bounded via the following simple lemma for cubes, several rather laboured versions of which appear in [5]: 
Lemma 4.1. Suppose we have $N$ variables $x_{i} \in[-1,1]$ with $\mathbb{E}_{i} x_{i}=s$ and $\mathbb{E}_{i} x_{i}^{2}=r$. Then we have the bound

$$
\mathbb{E}_{i} x_{i}^{3} \leq \frac{r(1+s)-\left(r^{2}+s^{2}\right)}{1-s}=g(r, s)
$$

Proof. Let $y_{i}=1-x_{i} \in[0,2]$, and observe see that $\mathbb{E}_{i} y_{i}=(1-s), \mathbb{E}_{i} y_{i}^{2}=$ $(1+r-2 s)$ as well as $\mathbb{E}_{i} y_{i}^{3}=(1+3 r-3 s)-\mathbb{E}_{i} x_{i}^{3}$. Bounding $\mathbb{E}_{i} y_{i}^{3}$ below by Cauchy-Schwarz

$$
\mathbb{E}_{i} y_{i}^{3} \geq \frac{\left(\mathbb{E}_{i} y_{i}^{2}\right)^{2}}{\mathbb{E}_{i} y_{i}}=\frac{(1+r-2 s)^{2}}{1-s}
$$

gives the desired result after rearranging.

It follows straightforwardly from Lemma 4.1 with $x_{i}$ replaced by $\mu_{e}-\delta_{e}$ and the preceding discussion that

$$
\mathbb{E}_{t}\left(p_{t}-q_{t}\right)^{2} \leq g(r, s)
$$

Inserting this bound via the Cauchy-Schwarz Inequality into (2), we find that

$$
32 K \geq 1+3 s+3 p-\sqrt{g(r, s)} .
$$

For the purpose of optimizing this expression, we observe that for $r \leq$ $(1+s) / 2$, the function $g(r, s)$ is decreasing in $r$. In general, we can bound $r$ (using nothing but the Cauchy-Schwarz Inequality and the definitions) by

$$
r \leq(2 \sqrt{s}+\sqrt{p})^{2}
$$

so we distinguish the cases $(2 \sqrt{s}+\sqrt{p})^{2} \geq(1+s) / 2$ and $(2 \sqrt{s}+\sqrt{p})^{2} \leq$ $(1+s) / 2$. In the first case, we can set $r=(1+s) / 2$, which implies that $(4)$ gives a lower bound for $p$ in terms of $s$, and we are left to find the minimum of the right-hand side of (3) as a function of the single variable $s$. In the second case, we set $r=(2 \sqrt{s}+\sqrt{p})^{2}$, and then minimize the right-hand side of (3) as a function of $r$ and $s$.

This is a question of seconds using a computer, and the minimum value thus obtained turns out to be $0.0217514 \ldots$, which lies between $1 / 46$ and $1 / 45$. 


\section{Thomason's upper bound for the number of monochromatic $K_{4} \mathrm{~S}$}

In 1989 Thomason [10] disproved a conjecture by Erdős which claimed that there are always at least the random number of monochromatic $K_{4}$ s in every 2-coloring of $K_{n}$. Even though there exists a wealth of counterexamples by now, this conjecture didn't seem quite so unreasonable back then. Indeed, the result is true if one replaces $K_{4}$ s by triangles (see [6]) or by ordinary 4-cycles. The initial construction Thomason gave to disprove Erdős's conjecture was rather obscurely phrased in terms of quadratic forms over a finite field (compare with the quadratic nature of Gowers's construction in Section $3)$. Equivalent and much clearer formulations have since appeared in [8] and [11].

It is interesting to note that the graphs constructed in these follow-up papers are quite similar in structure to the set constructed by Gowers which we described in Section 3. One takes a small example exhibiting a strong bias and then uses a product construction to produce a biased example of size growing asymptotically in $n$. In the case of Gowers's example we producted by long intervals, whereas Thomason uses a tensor product of graphs.

Definition. Given two graphs $J_{1}$ and $J_{2}$, let their tensor product $J_{1} \otimes J_{2}$ be the graph with vertex set $V\left(J_{1} \otimes J_{2}\right)=V\left(J_{1}\right) \times V\left(J_{2}\right)$. The edges of $J_{1} \otimes J_{2}$ are determined by $(v, w)\left(v^{\prime}, w^{\prime}\right) \in E\left(J_{1} \otimes J_{2}\right)$ if either $v v^{\prime} \in E\left(J_{1}\right)$ or $w w^{\prime} \in E\left(J_{2}\right)$ but not both.

(Many authors refer to this product as the Cartesian product of graphs. According to their definition, the tensor product requires both coordinates to be edges in the factor graphs, but we shall stick with Thomason's notation in order to minimize confusion.)

Note also that the tensor product is commutative and associative, and observe that if $J_{2}$ is the empty graph $\overline{K_{m}}$, then $J_{1} \otimes \overline{K_{m}}$ is the usual $m$-fold cover of $J_{1}$. We now rephrase the tensor product in terms of the balanced adjacency matrices of the graphs involved. We associate with $J$ the matrix $A(J)=(a(u, v))_{u, v \in V(J)}$ whose entries are indexed by the vertices of $J$ and are defined by $a(u, v)=-1$ if $u v \in E(J)$ and $a(u, v)=1$ otherwise. It is important to note that the diagonal entries of $A(J)$ are all equal to 1.

Definition. Given two square matrices $A=\left(a_{i j}\right)_{i, j=1}^{n}$ and $B=\left(b_{i j}\right)_{k, l=1}^{m}$, their tensor product $A \otimes B$ is defined to be the $n m \times n m$ square matrix with entries $(A \otimes B)_{(i, k)(j, l)}=a_{i j} b_{k l}$. 
It is straightforward to see that the matrix $A\left(J_{1} \otimes J_{2}\right)$ associated with the graph tensor product $J_{1} \otimes J_{2}$ is just the matrix tensor product $A\left(J_{1}\right) \otimes A\left(J_{2}\right)$.

We are now in a position to count the number of monochromatic $K_{4} \mathrm{~s}$ occurring in a given coloring of the complete graph $K_{n}$, or equivalently, the number of $K_{4}$ s occurring in a given graph $J \subseteq K_{n}$ and its complement, which we shall denote by $m_{K_{4}}(J)$. It is easy to check that $m_{K_{4}}(J)$ equals

$$
\sum_{u_{1}, \ldots, u_{4} \in V(J)} \prod_{i j \in E\left(K_{4}\right)}\left(1-a\left(u_{i}, u_{j}\right)\right)+\sum_{u_{1}, \ldots, u_{4} \in V(J)} \prod_{i j \in E\left(K_{4}\right)}\left(1+a\left(u_{i}, u_{j}\right)\right) .
$$

Writing

$$
\Psi(J, F)=|J|^{-4} \sum_{u_{1}, \ldots, u_{4} \in V(J)} \prod_{i j \in E(F)} a\left(u_{i}, u_{j}\right)
$$

for each spanning subgraph $F$ of $K_{4}$, we can rewrite $m_{K_{4}}(J)$ as

$$
2^{-5}|J|^{4}\left(1+O\left(|J|^{-1}\right)\right) \sum_{F \subseteq K_{4}} \Psi(J, F),
$$

where the sum is over all spanning subgraphs of $K_{4}$ with an even number of edges. It is clear from (5) that any graph $J$ with $\sum_{F \subseteq K_{4}} \Psi(J, F)<1$ will have fewer than the expected number of monochromatic $K_{4}$ s. Since $\Psi\left(\overline{K_{m}}, F\right)=1$ for all $F \subseteq K_{4}$, we see that in order to find a sequence of graphs with too few monochromatic $K_{4}$ s of order tending to infinity, it suffices to find a small graph $J$ with $\sum_{F \subset K_{4}} \Psi(J, F)<1$. One can then set $J_{m}=J \otimes \overline{K_{m}}$ to obtain the desired family.

As already noted in the seminal paper [3], which developed many of the fundamental notions we have described here, the function $\Psi(J, F)$ has the very useful property that it is multiplicative with respect to the tensor product of graphs defined above, in the sense that

$$
\Psi\left(J_{1} \otimes J_{2}, F\right)=\Psi\left(J_{1}, F\right) \Psi\left(J_{2}, F\right) .
$$

This allows one to compute the number of monochromatic $K_{4}$ s inside graph products with small factors very easily, since $\Psi(J, F)$ can be evaluated explicitly with little computational effort for small graphs $J$. (At this point we would like to draw the reader's attention to how $\Psi$ relates to the Fourier transform on $\mathbb{F}_{2}^{n !}$ !)

According to the computer investigations conducted in [11], the example which exhibits the largest relative bias amongst all tensor products of small graphs is the graph product $K_{4} \otimes M \otimes \overline{\left(K_{3} \otimes K_{3} \otimes \bar{K}_{2}\right)}$, where $M$ stands 
for the graph on 4 vertices with two non-adjacent edges. More precisely, computations result in the bound

$$
m_{K_{4}}\left(K_{4} \otimes M \otimes \overline{\left(K_{3} \otimes K_{3} \otimes \overline{K_{2}}\right)} \otimes \overline{K_{m}}\right)<\frac{1}{33}+o(1),
$$

where $o(1)$ stands for a quantity which tends to 0 as $m$ tends to infinity. It is observed in the final paragraph of [11] that it is possible to improve this construction by an absolutely tiny amount using a random perturbation.

\section{Concluding remarks}

Section 5 completed the fourth corner of the square defined by the axes graphs - sets and upper bound - lower bound which we have discussed in this article. It would be of great interest to close the gap between the upper and lower bound in both cases.

While we have pointed out some tentative analogies between the world of graphs and sets, their exact nature remains somewhat elusive. In particular, Gowers's set is uniform yet contains the wrong number of 4-APs. In the world of graphs, a uniform (that is, quasirandom) graph contains the correct number of $K_{4} \mathrm{~s}$ and will therefore be of no use in constructing a bad example. In view of this breakdown of analogies, it seems likely that in order to fully understand Thomason's constructions, one needs to instead consider notions of uniformity which have been developed in the context of hypergraphs.

\section{Acknowledgements}

The author would like to thank Tim Gowers for making the preprint [7] available, Andrew Thomason for several helpful discussions and the anonymous referees for useful additions to the bibliography.

\section{References}

[1] P. Cameron, J. Cilleruelo and O. Serra. On monochromatic solutions of equations in groups. Rev. Mat. Iberoam., 23:385-395, 2007. MR2351139

[2] S. Butler, K. Costello and R. Graham. Finding patterns avoiding many monochromatic constellations. To appear, Experiment. Math., 2010. 
[3] F. R. K. Chung and R. Graham. Quasirandom set systems. J. Amer. Math. Soc., 4(1):151-196, 1991. MR1077279

[4] B. Datskovsky. On the number of monochromatic Schur triples. Adv. Appl. Math., 31:193-198, 2003. MR1985828

[5] G. Giraud. Sur le problème de Goodman pour les quadrangles et la majoration des nombres de Ramsey. J. Combin. Theory Ser. B, 27(3):237253, 1979. MR0554292

[6] A. W. Goodman. On sets of acquaintances and strangers at any party. Amer. Math. Monthly, 66(778-783), 1959. MR0107610

[7] W. T. Gowers. Two examples in additive combinatorics. Submitted, 2007.

[8] C. Jagger, P. Šťvíček and A. Thomason. Multiplicities of subgraphs. Combinatorica, 16(1):123-141, 1996. MR1394515

[9] P. Parillo, A. Robertson and D. Saracino. On the asymptotic minimum number of monochromatic 3-term arithmetic progressions. J. Combin. Theory Ser. A, 115:185-192, 2008. MR2378863

[10] A. Thomason. A disproof of a conjecture of Erdős in Ramsey theory. J. London Math. Soc., 39(2):246-255, 1989. MR0991659

[11] A. Thomason. Graph products and monochromatic multiplicities. Combinatorica, 17(1):125-134, 1997. MR1466580

J. WOLF

Rutgers, The State University of New Jersey

Department of Mathematics

110 FrelinghuYsen RD.

PISCATAWAY, NJ 08854

USA

E-mail address: julia.wolf@cantab.net

Received December 3, 2009 